\title{
Quantitative Film Thickness Measurement Using Scintillator/Photomultiplier Backscattered Electron Detectors. Possible or Not?
}

Pierre Hovington, Marin Lagacé, Pierre Noël, *Hydro-Quebec Research Institute, 1800 boul. Lionel-Boulet, Varennes, Québec, Canada, J3X 1S1

Scintillator/photomultiplicator backscattered electron detectors (BSE) are now commonly used in scanning electron microscopy (SEM) since they have sufficient sensitivity to detect low energy backscattered electrons at high scan rates (i.e. TV rate). These detectors are often the main source of imaging in SEMs under variable pressure operating mode. Two scintillating materials are mainly used to convert electron signal to light: high-Z powders and plastic scintillators [1]. The most commonly used are high- $Z$ powders coated on a conductive layer (Al or other) to remove charging. However, those detectors have very rough surfaces. These rough surfaces combined with the high- $Z$ material can produce a large amount of type III BSEs. In addition, the number of BSEs hitting the high-Z scintillating material depends on the angular distribution of the BSEs emitted from the sample. Taking this into consideration along with the fact that the samples being analyzed may have high surface roughness, we have evaluated the possibility of obtaining quantitative information from the BSE signal.

In our investigation, we have used an $\mathrm{Al}$ foil (approximately $12 \mu \mathrm{m}$ in thickness) coated with a mixture of polymer and conductive carbon $\left(\rho=1.12 \mathrm{~g} / \mathrm{cm}^{3}\right)$. This film is often used as a current collector in battery applications. One of the major concerns for this type of application is to always have at the very least $0.5 \mu \mathrm{m}$ of protective carbon. A cross-section can be used to directly measure coating thickness (cf. Figure 1). However, this process can only cover a very small area of the sample. Furthermore, getting a properly polished cross-section for this type of material is very labour intensive. Since the coating is quite thin $(0.5 \mu \mathrm{m}$ to $2 \mu \mathrm{m})$, it is possible to observe the sample in plan section and to optimized the acceleration voltage in order to obtain BSE contrast from the variations in the coating thickness (cf. Figure 2). Using Monte Carlo simulation [2], it is possible to convert greyscale micrographs into thickness images using a calibration curve (cf. Figure 3) and adjusting the micrograph's color palette to reflect this curve (cf. Figure 4).

In order to verify the computed thickness in plan section, we carried out a series of measurements in cross-section on the same region. The histogram of both the computed and measured thickness distributions are presented in Figures 5 and 6 . The computed results (plan section) seem to slightly overestimate the measured ones (cross-section) by approximately $200 \mathrm{~nm}$. This could be caused by inaccurate coating density data or by the non-linearity of the BSE output gain. Further analysis will be done in order to clarify this point.

In conclusion, scintillator/photomultiplicator BSE detectors can be used in order to get a first approximation of the thickness of a carbon/polymer over Al thin film. Taking into account the surface roughness of these types of detectors, these results are quite encouraging but special care must be taken to get the proper calibration curve.

References:

[1] www.carroll-ramsey.com/scintillatorapps.htm\#10

[2] Hovington, P. et al., (1997), Scanning Vol. 19, pp. 1-14

[3] This work was sponsored by Hydro-Quebec Research Institute. 

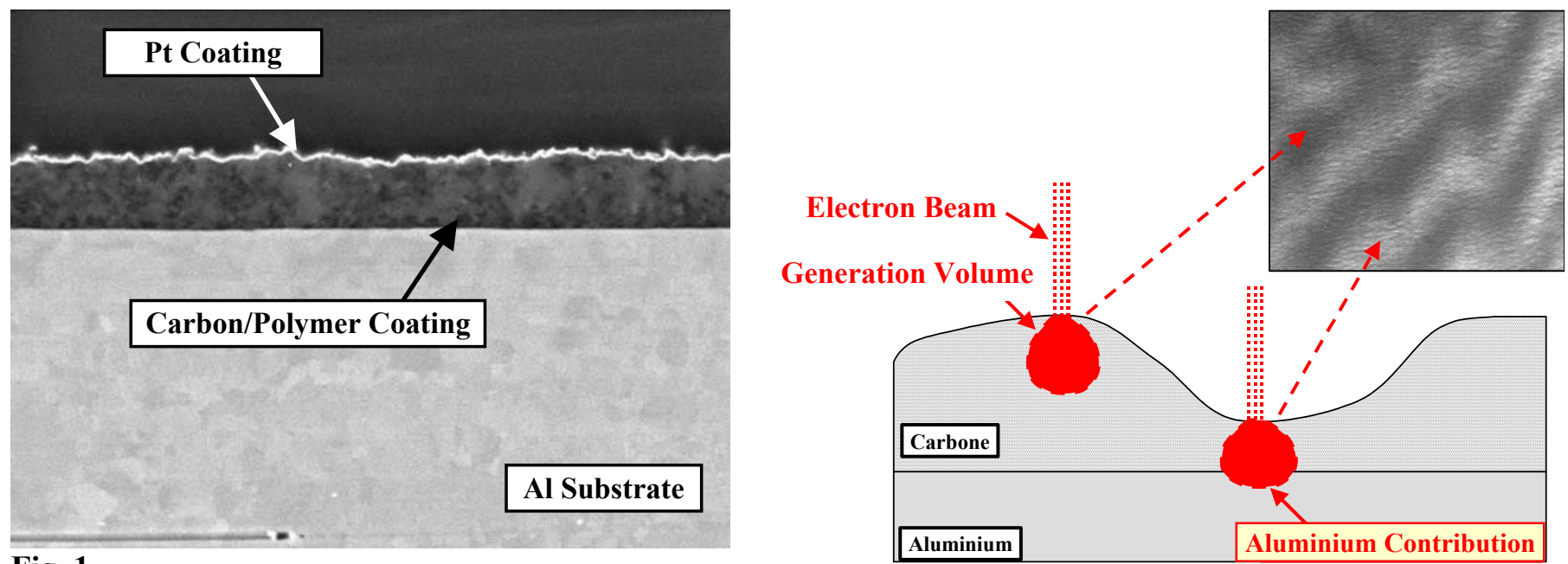

Fig. 1

Fig. 2

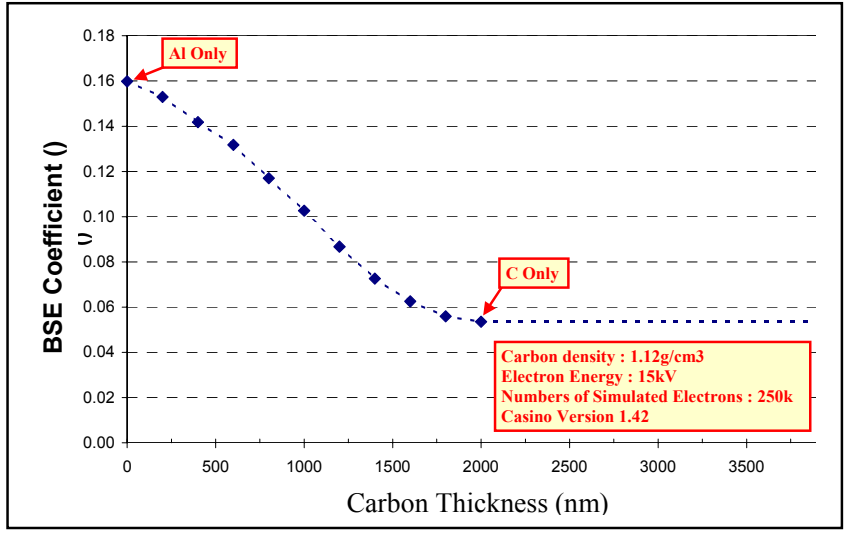

Fig. 3

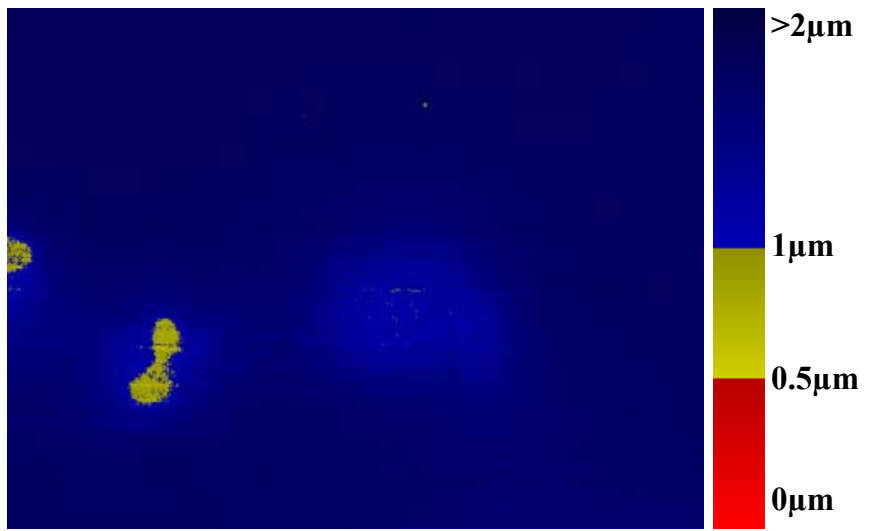

Fig. 4

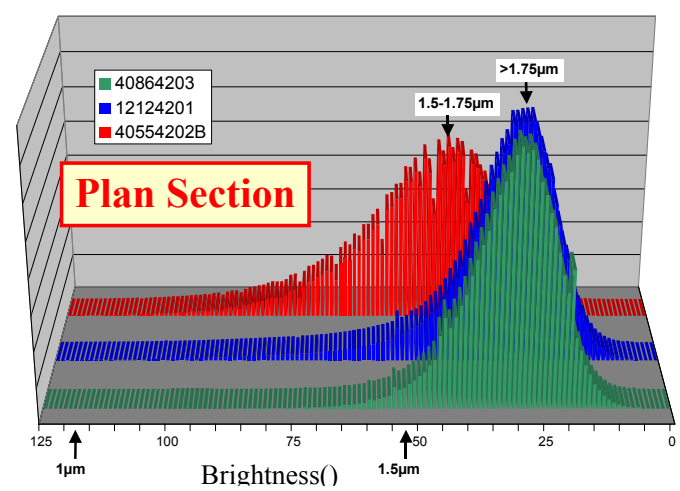

Fig. 5

Fig. 6

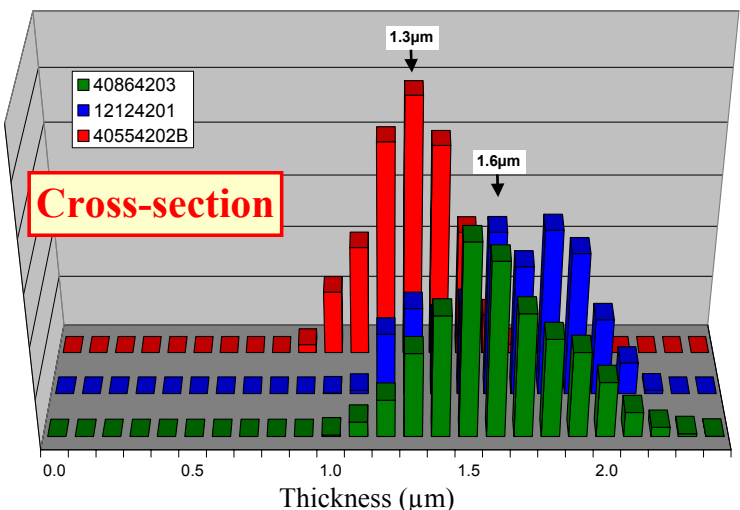

Figure 1: Cross-section view of the carbon/polymer coating over an Al substrate.

Figure 2: Schematic representation showing contrast in the BSE micrographs as a result of changes in coating thickness.

Figure 3: Calibration curve used to convert BSE contrast to film thickness. The results where computed using Casino version 2.42 (windows version).

Figure 4: BSE micrograph color coded for thickness using the calibration curve shown in Figure 3.

Figure 5: Thickness computed using BSE micrographs in a plan view.

Figure 6: Thickness measured using BSE micrographs in a cross-section. 